\title{
Amygdala Surface Modeling with Weighted Spherical Harmonics
}

\author{
University of Wisconsin, Madison \\ Department of Biostatistics and Medical Informatics \\ Technical Report 202
}

\author{
Moo K. Chung ${ }^{12}$, Brendon, M. Nacewicz ${ }^{2}$, Shubing Wang ${ }^{1}$, \\ Kim M. Dalton ${ }^{2}$, Seth Pollak ${ }^{3}$, Richard J. Davidson ${ }^{23}$ \\ ${ }^{1}$ Department of Statistics, Biostatistics and Medical Informatics \\ ${ }^{2}$ Waisman Laboratory for Brain Imaging and Behavior \\ ${ }^{3}$ Department of Psychology and Psychiatry \\ University of Wisconsin, Madison, WI 53706, USA \\ mkchung@wisc.edu
}

\begin{abstract}
Although there are numerous publications on amygdala volumetry, so far there has not been many studies on modeling local amygdala surface shape variations in a rigorous framework. This paper present a systematic framework for modeling local amygdala shape. Using a novel surface flattening technique, we obtain a smooth mapping from the amygdala surface to a sphere. Then taking the spherical coordinates as a reference frame, amygdala surfaces are parameterized as a weighted linear combination of smooth basis functions using the recently developed weighted spherical harmonic representation. This new representation is used for parameterizing, smoothing and nonlinearly registering a group of amygdala surfaces. The methodology has been applied in detecting abnormal local shape variations in 23 autistic subjects compared against 24 normal controls. We did not detect any statistically significant abnormal amygdala shape variations in autistic subjects. The complete amygdala surface modeling codes used in this study is available at http://www.stat.wisc.edu/ mchung/research/amygdala.
\end{abstract}

\section{Introduction}

Amygdala is an important brain substructure that has been implicated in abnormal functional impairment in autism [7] [14]. Since the abnormal structure might be the cause of the functional impairment, there have been many studies on amygdala volumetry in autism. However, most amygdala volumetry results are somewhat inconsistent [2] [16] [10] [14]. The previous studies traced the amygdalae manually and by counting the number of voxels within the region of interest (ROI), the total volume of the amygdalae were estimated. The limitation of the traditional ROI-based volumetry is that it can not determine if the 
volume difference is spread all over the ROI or localized within specific regions of the ROI.

In this paper, we present a new framework for addressing the problem of local amygdala shape analysis using the recently developed weighted spherical harmonic representation [5]. The weighted spherical harmonic representation formulates surface parameterization, filtering and nonlinear surface registration in a unified Hilbert space framework. Since the proposed method requires a surface flattening to a sphere, we have developed a new and very fast surface flattening technique based on the equilibrium state of heat diffusion. By tracing the geodesic path of heat equilibrium state from a heat source (amygdala) to a heat sink (sphere), we obtain a smooth spherical mapping. Solving an isotropic heat equation in a 3D image volume is computationally trivial, so our proposed method offers a much simpler numerical implementation than previous surface flattening techniques such as conformal mappings [1] [9] [11], quasi-isometric mappings [17] and area preserving mappings [4]. These flattening methods are not trivial to implement and computationally insensitive. Once we obtain the weighted spherical harmonic representation of amygdalae, the group difference between 23 autistic and 24 control subjects is statistically tested using the Hotelling's $T^{2}$ statistic on the estimated surface coordinates.

\section{Methods}

High resolution anatomical magnetic resonance images (MRI) were obtained using a 3-Tesla GE SIGNA scanner with a quadrature head coil. Details on image acquisition parameters are given in [14]. MRIs are reoriented to the pathological plane [6] for optimal comparison with anatomical atlases. Manual segmentation was done by an expert and the reliability of the manual segmentation was validated by two raters on 10 amygdale resulting in interclass correlation of 0.95 and the intersection over the union of 0.84 [14]. Once binary segmentation is obtained, the marching cubes algorithm was used to extract the boundary of amygdale. Afterwards, we we flattened the amaygada surfaces using the proposed method.

\subsection{Surface Flattening via Diffusion}

Given an amygdala binary segmentation $\mathcal{M}_{a}$, we put a larger sphere $\mathcal{M}_{s}$ that encloses the amygala (Figure 1 left). The amygdala is assigned the value 1 while the enclosing sphere is assigned the value -1, i.e.

$$
f\left(\mathcal{M}_{a}, \sigma\right)=1 \text { and } f\left(\mathcal{M}_{s}, \sigma\right)=-1
$$

for all $\sigma$. The parameter $\sigma$ denotes the diffusion time. The amygdala and the sphere serve as a heat source and a heat sink respectively. Then we solve an isotropic diffusion equation

$$
\frac{\partial f}{\partial \sigma}=\Delta f
$$




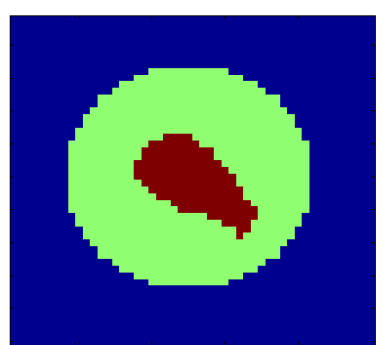

Boundary condition

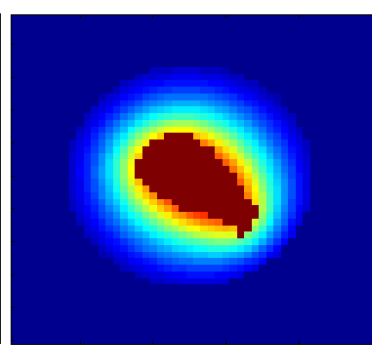

Equilibrium state

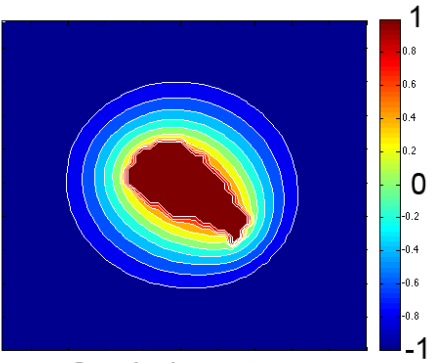

Geodesic contour

Fig. 1. The diffusion equation with a heat source (amygdala) and a heat sink (enclosing sphere). After sufficient number of iterations, the equilibrium state is reached. By tracing the geodesic path from the heat source to the heat sink using the geodesic contour, we obtain a smooth spherical mapping.

with the given boundary condition. $\Delta$ is the 3D Laplacian. After sufficiently enough time, the solution reaches the heat equilibrium state where the additional diffusion does not make any change in the heat distribution (Figure 1 middle). The heat equilibrium state can be also obtained by letting $\frac{\partial f}{\partial \sigma}=0$ and solving for the Laplace equation

$$
\Delta f=0
$$

with the same boundary condition. The resulting equilibrium state is given in Figure 1 (middle).

Once we obtained the equilibrium state, we trace the geodesic path from the heat source to the heat sink for every mesh vertices on the isosurface of the amygdala. The trajectory of the geodesic path provides a smooth mapping from the amygdala surface to the sphere. The geodesic path can be traced by following the gradient of the equilibrium state but this requires solving an additional system of differential equations. So we have avoided using the equilibrium gradient. Instead we have constructed numerous geodesic contours that correspond to the level set of the equilibrium state (Figure 1 right. Then the geodesic path is constructed by finding the shortest distance from one contour to the next and connecting them together. This is done in an iterative fashion as shown in Figure 2 , where five contours corresponding to the values $0.6,0.2,-0.2,-0.6,-1.0$ are used to flatten the amygdala surface. Once we obtained the spherical mapping, we can project the Euler angles $(\theta, \varphi)$ onto the amygdala surface (Figure 3) and the Euler angles serve as the underlying parameterization for the weighted spherical harmonic modeling.

\subsection{Weighted Spherical Harmonics}

Since the technical detail and numerical implementation for the weighted spherical harmonic modeling is given in [5], we will only briefly describe the basic 


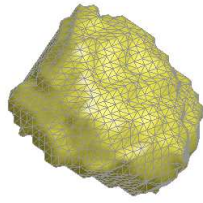

1.0

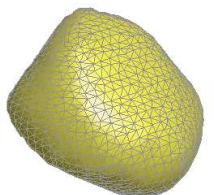

0.6

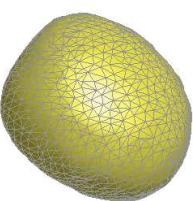

0.2

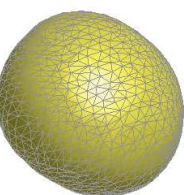

$-0.2$

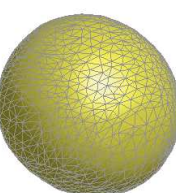

$-0.6$

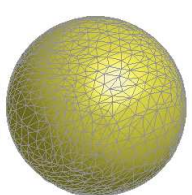

$-1.0$

Fig. 2. Amygala surface flattening process following the geodesic path of the equilibrium state of heat diffusion. The numbers corresponds to the geodesic contours. For simple shapes like amygda, 5 to 10 contours are sufficient for tracing the geodesic path.

idea here. The weighted spherical harmonic representation fixes the Gibbs phenomenon (ringing effects) associated with the traditional Fourier descriptors and spherical harmonic representation [4] [8] [9] [12] [15] by weighting the series expansion with exponential weights. The exponential weights make the representation converges faster and reduces the amount of wiggling. If surface coordinates are abruptly changing or their derivatives are discontinuous, the Gibbs phenomenon will severely distort the surface shape as shown in Figure 4, where a cube is reconstructed with both the traditional $(k=42, \sigma=0)$ and the new weighted spherical harmonics $(k=42, \sigma=0.001)$. The weighted version has less ringing effects.

From the surface flattening, the mesh coordinates for the amygdala surface $\partial \mathcal{M}_{a}$ can be parameterized by the Euler angles $\theta \in[0, \pi], \varphi \in[0,2 \pi)$ associated with the unit sphere as

$$
p(\theta, \varphi)=\left(p_{1}(\theta, \varphi), p_{2}(\theta, \varphi), p_{2}(\theta, \varphi)\right)^{\prime} .
$$

See Figure 3 for how the Euler angles are used to parameterize the amygdala surface. The weighted spherical harmonic representation of the coordinates is then given by

$$
p(\theta, \varphi)=\sum_{l=0}^{k} \sum_{m=-l}^{l} e^{-l(l+1) \sigma} f_{l m} Y_{l m}(\theta, \varphi),
$$

where

$$
f_{l m}=\int_{\theta=0}^{\pi} \int_{\varphi=0}^{2 \pi} p(\theta, \varphi) Y_{l m}(\theta, \varphi) \sin \theta d \theta d \varphi .
$$

are the Fourier coefficient vectors and $Y_{l m}$ are spherical harmonics of degree $l$ and order $m$. The coefficients $f_{l m}$ are estimated by one degree at a time in the least squares fashion using the recently developed iterative residual fitting algorithm. We have used the 15-th degree representation for this study (Figure $5)$.

Once amgydala surfaces are represented with the weighted spherical harmonics, the spherical harmonic correspondence can be used to nonlinearly align all 47 amygdale surfaces. The average left and right amygdala templates are 

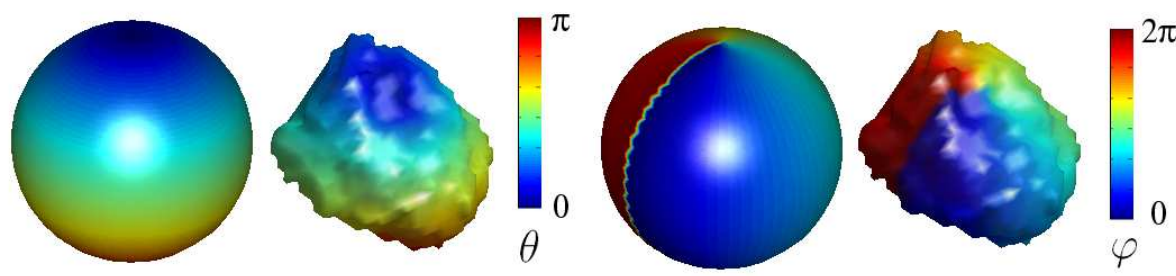

Fig. 3. Amygdala surface parameterization using the Euler angles $(\theta, \varphi)$. The point $\theta=0$ corresponds to the north pole of a unit sphere. The parameterization is needed for the weighted spherical harmonic modeling.

constructed by averaging the Fourier coefficients of all 24 control subjects. The smooth surfaces in Figure 6 are the constructed average templates. The template surfaces serve as the reference coordinates for projecting the subsequent statistical parametric maps.

\subsection{Comparing Two Groups of Surfaces}

Let $i$ be the group index and $j$ be the subject index. Let $n_{i}$ be the sample size in the $i$-th group. For convenience, let the first group to be normal controls $\left(n_{1}=\right.$ $24)$ and the second group to be autistic $\left(n_{2}=23\right)$. We propose the following stochastic model on the weighted spherical harmonics. For surface coordinates $p_{i j}=\left(p_{i j}^{1}, p_{i j}^{2}, p_{i j}^{3}\right)^{\prime}$, we model

$$
p_{i j}(\theta, \varphi)=\sum_{l=0}^{k} \sum_{m=-l}^{l} e^{-l(l+1) \sigma} \mu_{l m}^{i j} Y_{l m}(\theta, \varphi)+\Sigma^{1 / 2}(\theta, \varphi) \epsilon_{i j}(\theta, \varphi),
$$

where $\mu_{l m}^{i j}$ are unknown Fourier coefficient vectors, $\Sigma$ is the covariance matrix, which allows the spatial dependence among $p_{i 1}, p_{i 2}, p_{i 3}$, and $\epsilon_{i j}$ are independent and identically distributed Gaussian random vector field. A similar stochastic modeling approach has been used in [13] where the canonical expansion of a Gaussian random field is used to model deformation vector fields. Then we test the following null $H_{0}$ and alternate $H_{1}$ hypotheses:

$$
\begin{aligned}
& H_{0}: \sum_{l=0}^{k} \sum_{m=-l}^{l} e^{-l(l+1) \sigma} \mu_{l m}^{1 j} Y_{l m}(\theta, \varphi)=\sum_{l=0}^{k} \sum_{m=-l}^{l} e^{-l(l+1) \sigma} \mu_{l m}^{2 j} Y_{l m}(\theta, \varphi) \\
& \text { vs. } \\
& H_{1}: \sum_{l=0}^{k} \sum_{m=-l}^{l} e^{-l(l+1) \sigma} \mu_{l m}^{1 j} Y_{l m}(\theta, \varphi) \neq \sum_{l=0}^{k} \sum_{m=-l}^{l} e^{-l(l+1) \sigma} \mu_{l m}^{2 j} Y_{l m}(\theta, \varphi) .
\end{aligned}
$$

The unknown group mean for the $i$-th group is estimated by

$$
\overline{p_{i}}=\frac{1}{n_{i}} \sum_{j=1}^{n_{i}} p_{i j}
$$




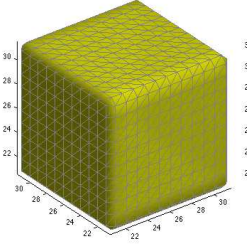

Cube

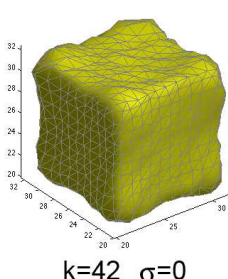

$k=42 \quad \sigma=0$
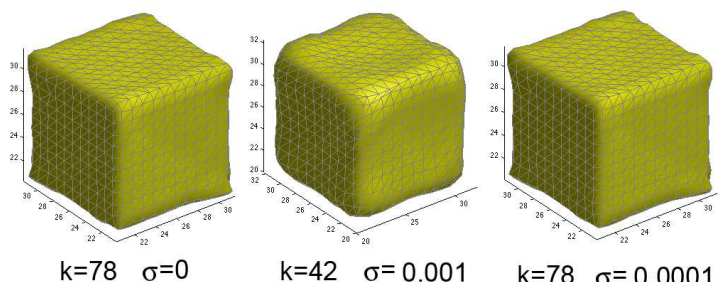

Fig. 4. The severe Gibbs phenomenon shown in the traditional spherical harmonic model of a cube $(\sigma=0)$ for degrees $k=42,78$. The weighted versions can reduce the Gibbs phenomenon in the representation by introducing small weights corresponding to $\sigma=0.001,0.0001$.

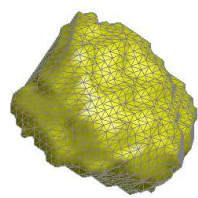

Original

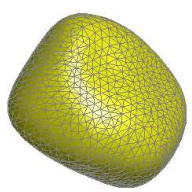

5

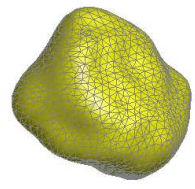

10

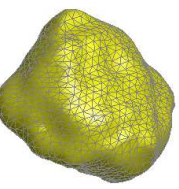

15

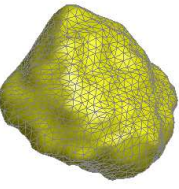

20

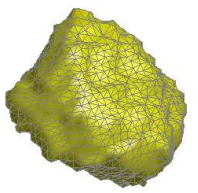

60

Fig. 5. The spherical harmonic modeling of a left amygdala surface with various degrees. We have chosen degree 15 representation in this study.

The group mean difference vector $\bar{p}_{2}-\bar{p}_{1}$ is shown as white arrows in Figure 6 . The group difference is only shown in the regions with $P$-value $<0.01$ for better visualization. The direction of white arrows is where the mean control surface should be moved to match with the mean amygdala surface. The significance of the group difference can be tested using the Hotelling's $T^{2}$ statistic given by

$$
H(\theta, \varphi)=\frac{n_{1} n_{2}\left(n_{1}+n_{2}-4\right)}{3\left(n_{1}+n_{2}\right)\left(n_{1}+n_{2}-2\right)}\left(\bar{p}_{2}-\bar{p}_{1}\right)^{\prime} \widehat{\Sigma}^{-1}\left(\bar{p}_{2}-\bar{p}_{1}\right),
$$

where

$$
\widehat{\Sigma}=\frac{1}{n_{1}+n_{2}-2}\left[\sum_{j=1}^{n_{1}}\left(p_{1 j}-\bar{p}_{1}\right)\left(p_{1 j}-\bar{p}_{1}\right)^{\prime}+\sum_{j=1}^{n_{2}}\left(p_{2 j}-\bar{p}_{1}\right)\left(p_{2 j}-\bar{p}_{2}\right)^{\prime}\right] .
$$

$H(\theta, \varphi)$ is distributed as a $F$-statistic with 3 and $n_{1}+n_{2}-4$ degrees of freedom.

\section{Results}

The volumes for control subjects $\left(n_{1}=24\right)$ are left $1883 \pm 176 \mathrm{~mm}^{3}$, right $1874 \pm$ $172 \mathrm{~mm}^{3}$. The volumes for autistic subjects $\left(n_{2}=23\right)$ are left $1859 \pm 182 \mathrm{~mm}^{3}$, 


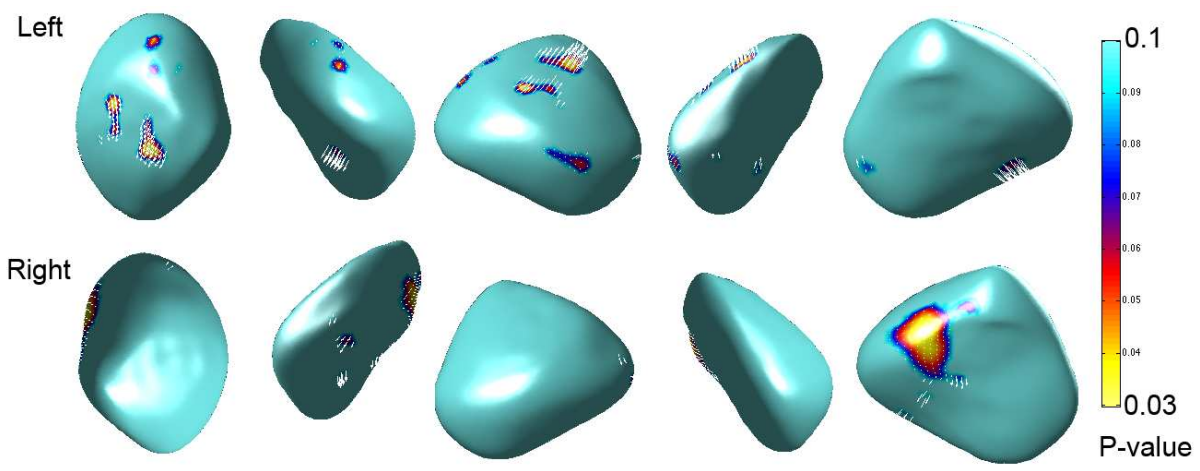

Fig. 6. The $P$-value of Hotelling's $T^{2}$ statistic projected onto the average amydala template constructed from 24 control subjects. The white arrows show the direction where the average control surface should move to match the average autistic surface.

right $1862 \pm 181 \mathrm{~mm}^{3}$. The volume difference between the groups are not statistically significant $(P$-value $=0.64$ for left and 0.81 for right $)$.

From the ROI-based volumetry, it is not clear if the local shape difference is still present within amygdala. So we have performed the Hotelling's $T^{2}$ test the average surface template at each mesh vertex. The resulting $P$-value is given in Figure 6 . The minimum $P$-value is 0.03 for both the left and the right amygdale. Although, at a fixed point, this is sufficiently low $P$-value to be taken as a significant signal, the result will not pass the multiple comparison correction based on the random field theory [18] or false discovery rate (FDR) [3]. So we conclude that there is no abnormal local amygdala shape difference in autism.

\section{Conclusions}

The paper developed a unified framework for quantifying a population of amygdala surfaces. Our main contribution is the new amygdala surface flattening technique that utilizes the idea of the geodesic path of heat equilibrium. The proposed flattening technique is simple enough to be applied to various applications. Using the spherical mapping established from the new flattening technique, we have applied the recently developed weighted spherical harmonic representation to parameterize, to register amygdala surfaces, and to detect local shape difference. We found no statistically significant local amygdala shape difference between autism and control. The complete compute codes used for this study is available at http://www. stat.wisc.edu/ mchung/research/amygdala.

\section{References}

1. S. Angenent, S. Hacker, A. Tannenbaum, and R. Kikinis. On the laplace-beltrami operator and brain surface flattening. IEEE Transactions on Medical Imaging, 18:700-711, 1999. 
2. E.H. Aylward, N.J Minshew, G. Goldstein, N.A. Honeycutt, A.M. Augustine, K.O. Yates, P.E. Bartra, and G.D. Pearlson. Mri volumes of amygdala and hippocampus in nonmentally retarded autistic adolescents and adults. Neurology, 53:2145-2150, 1999.

3. Y. Benjamini and Y. Hochberg. Controlling the false discovery rate: a practical and powerful approach to multiple testing. J. R. Stat. Soc, Ser. B, 57:289-300, 1995.

4. C. Brechbuhler, G. Gerig, and O. Kubler. Parametrization of closed surfaces for 3d shape description. Computer Vision and Image Understanding, 61:154-170, 1995.

5. M.K. Chung, L. Shen Dalton, K.M., A.C. Evans, and R.J. Davidson. Weighted fourier representation and its application to quantifying the amount of gray matter. IEEE transactions on medical imaging, 26:566-581, 2007.

6. A. Convit, P McHugh, O.T. Wolf, M.J. de Leon, M. Bobinkski, S. De Santi, A. Roche, and W. Tsui. Mri volume of the amygdala: a reliable method allowing separation from the hippocampal formation. Psychiatry Res., 90:113-123, 1999.

7. K.M. Dalton, B.M. Nacewicz, T. Johnstone, H.S. Schaefer, M.A. Gernsbacher, H.H. Goldsmith, A.L. Alexander, and R.J. Davidson. Gaze fixation and the neural circuitry of face processing in autism. Nature Neuroscience, 8:519-526, 2005.

8. G. Gerig, M. Styner, D. Jones, D. Weinberger, and J. Lieberman. Shape analysis of brain ventricles using spharm. In MMBIA, pages 171-178, 2001.

9. X. Gu, Y.L. Wang, T.F. Chan, T.M. Thompson, and S.T. Yau. Genus zero surface conformal mapping and its application to brain surface mapping. IEEE Transactions on Medical Imaging, 23:1-10, 2004.

10. M.M. Haznedar, M.S. Buchsbaum, T.C. Wei, P.R. Hof, C Cartwright, and E. Bienstock, C.A. Hollander. Limbic circuitry in patients with autism spectrum disorders studied with positron emission tomography and magnetic resonance imaging. American Journal of Psychiatry, 157:1994-2001, 2000.

11. M. K. Hurdal and K. Stephenson. Cortical cartography using the discrete conformal approach of circle packings. NeuroImage, 23:S119S128, 2004.

12. A. Kelemen, G. Szekely, and G. Gerig. Elastic model-based segmentation of 3-d neuroradiological data sets. IEEE Transactions on Medical Imaging, 18:828-839, 1999.

13. M.I. Miller, A. Banerjee, G.E. Christensen, S.C. Joshi, N. Khaneja, U. Grenander, and L. Matejic. Statistical methods in computational anatomy. Statistical Methods in Medical Research, 6:267-299, 1997.

14. B.M. Nacewicz, K.M. Dalton, T. Johnstone, M.T. Long, E.M. McAuliff, T.R. Oakes, A.L Alexander, and R.J. Davidson. Amygdala volume and nonverbal social impairment in adolescent and adult males with autism. Arch. Gen. Psychiatry., 63:1417-1428, 2006.

15. L. Shen, J. Ford, F. Makedon, and A. Saykin. surface-based approach for classification of 3d neuroanatomical structures. Intelligent Data Analysis, 8:519-542, 2004 .

16. B.F. Sparks, S.D. Friedman, D.W. Shaw, E.H. Aylward, D. Echelard, A.A. Artru, K.R. Maravilla, J.N. Giedd, J. Munson, G. Dawson, and S.R. Dager. Brain structural abnormalities in young children with autism spectrum disorder. Neurology, 59:184-192, 2002.

17. B. Timsari and R. Leahy. An optimization method for creating semi-isometric flat maps of the cerebral cortex. In The Proceedings of SPIE, Medical Imaging, 2000.

18. K.J. Worsley, S. Marrett, P. Neelin, A.C. Vandal, K.J. Friston, and A.C. Evans. A unified statistical approach for determining significant signals in images of cerebral activation. Human Brain Mapping, 4:58-73, 1996. 